\title{
Deep learning for real-time, automatic, and scanner-adapted prostate (zone) segmentation of transrectal ultrasound, for example, magnetic resonance imaging-transrectal ultrasound fusion prostate biopsy
}

Citation for published version (APA):

van Sloun, R. J. G., Wildeboer, R. R., Mannaerts, C. K., Postema, A. W., Gayet, M., Beerlage, H. P., Salomon, G., Wijkstra, H., \& Mischi, M. (2021). Deep learning for real-time, automatic, and scanner-adapted prostate (zone) segmentation of transrectal ultrasound, for example, magnetic resonance imaging-transrectal ultrasound fusion prostate biopsy. European Urology Focus, 7(1), 78-85. https://doi.org/10.1016/j.euf.2019.04.009

\section{Document license:}

TAVERNE

DOI:

10.1016/j.euf.2019.04.009

Document status and date:

Published: 01/01/2021

\section{Document Version:}

Publisher's PDF, also known as Version of Record (includes final page, issue and volume numbers)

\section{Please check the document version of this publication:}

- A submitted manuscript is the version of the article upon submission and before peer-review. There can be important differences between the submitted version and the official published version of record. People interested in the research are advised to contact the author for the final version of the publication, or visit the $\mathrm{DOI}$ to the publisher's website.

- The final author version and the galley proof are versions of the publication after peer review.

- The final published version features the final layout of the paper including the volume, issue and page numbers.

Link to publication

\footnotetext{
General rights

- You may freely distribute the URL identifying the publication in the public portal. follow below link for the End User Agreement:

www.tue.nl/taverne

\author{
Take down policy \\ If you believe that this document breaches copyright please contact us at: \\ openaccess@tue.nl \\ providing details and we will investigate your claim.
}

Copyright and moral rights for the publications made accessible in the public portal are retained by the authors and/or other copyright owners and it is a condition of accessing publications that users recognise and abide by the legal requirements associated with these rights.

- Users may download and print one copy of any publication from the public portal for the purpose of private study or research.

- You may not further distribute the material or use it for any profit-making activity or commercial gain

If the publication is distributed under the terms of Article 25fa of the Dutch Copyright Act, indicated by the "Taverne" license above, please 


\title{
Deep Learning for Real-time, Automatic, and Scanner-adapted Prostate (Zone) Segmentation of Transrectal Ultrasound, for Example, Magnetic Resonance Imaging-transrectal Ultrasound Fusion Prostate Biopsy
}

\author{
Ruud J.G. van Sloun ${ }^{a, *}$, Rogier R. Wildeboer ${ }^{a}$, Christophe K. Mannaerts ${ }^{b}$, Arnoud W. Postema ${ }^{b}$, \\ Maudy Gayet ${ }^{c}$, Harrie P. Beerlage ${ }^{a, b}$, Georg Salomon ${ }^{d}$, Hessel Wijkstra ${ }^{a, b}$, Massimo Mischi $^{a}$ \\ ${ }^{\mathrm{a}}$ Laboratory of Biomedical Diagnostics, Department of Electrical Engineering, Eindhoven University of Technology, Eindhoven, The Netherlands; ${ }^{\mathrm{b}}$ Department \\ of Urology, Amsterdam University Medical Centers, University of Amsterdam, Amsterdam, The Netherlands; ${ }^{\mathrm{c}}$ Department of Urology, Jeroen Bosch Hospital, \\ 's-Hertogenbosch, The Netherlands; ${ }^{\mathrm{d}}$ Martini Klinik-Prostate Cancer Center, University Hospital Hamburg Eppendorf, Hamburg, Germany
}

\section{Article info}

\section{Article history:}

Accepted April 10, 2019

Associate Editor

:Derya Tilki

\section{Keywords:}

Deep learning

Prostate cancer

Segmentation

Ultrasound

magnetic resonance imagingtransrectal ultrasound fusion biopsy

\begin{abstract}
Background: Although recent advances in multiparametric magnetic resonance imaging (MRI) led to an increase in MRI-transrectal ultrasound (TRUS) fusion prostate biopsies, these are time consuming, laborious, and costly. Introduction of deep-learning approach would improve prostate segmentation.

Objective: To exploit deep learning to perform automatic, real-time prostate (zone) segmentation on TRUS images from different scanners.

Design, setting, and participants: Three datasets with TRUS images were collected at different institutions, using an iU22 (Philips Healthcare, Bothell, WA, USA), a Pro Focus 2202a (BK Medical), and an Aixplorer (SuperSonic Imagine, Aix-en-Provence, France) ultrasound scanner. The datasets contained 436 images from 181 men.

Outcome measurements and statistical analysis: Manual delineations from an expert panel were used as ground truth. The (zonal) segmentation performance was evaluated in terms of the pixel-wise accuracy, Jaccard index, and Hausdorff distance.

Results and limitations: The developed deep-learning approach was demonstrated to significantly improve prostate segmentation compared with a conventional automated technique, reaching median accuracy of 98\% (95\% confidence interval 95-99\%), a Jaccard index of $0.93(0.80-0.96)$, and a Hausdorff distance of $3.0(1.3-8.7) \mathrm{mm}$. Zonal segmentation yielded pixel-wise accuracy of $97 \%$ (95-99\%) and 98\% (96-99\%) for the peripheral and transition zones, respectively. Supervised domain adaptation resulted in retainment of high performance when applied to images from different ultrasound scanners $(p>0.05)$. Moreover, the algorithm's assessment of its own segmentation performance showed a strong correlation with the actual segmentation performance (Pearson's correlation 0.72, $p<0.001$ ), indicating that possible incorrect segmentations can be identified swiftly. Conclusions: Fusion-guided prostate biopsies, targeting suspicious lesions on MRI using TRUS are increasingly performed. The requirement for (semi)manual prostate delineation places a substantial burden on clinicians. Deep learning provides a means for fast and accurate (zonal) prostate segmentation of TRUS images that translates to different scanners.

* Corresponding author. Laboratory of Biomedical Diagnostics, Eindhoven University of Technology, PO-Box 513m, 5600 MB Eindhoven, The Netherlands. Tel.: +31 40-2473821.

E-mail address: r.j.g.v.sloun@tue.nl (Ruud J.G. van Sloun).
\end{abstract}


Patient summary: Artificial intelligence for automatic delineation of the prostate on ultrasound was shown to be reliable and applicable to different scanners. This method can, for example, be applied to speed up, and possibly improve, guided prostate biopsies using magnetic resonance imaging-transrectal ultrasound fusion.

(c) 2019 European Association of Urology. Published by Elsevier B.V. All rights

reserved.

\section{Introduction}

Owing to recent advances in multiparametric magnetic resonance imaging (MRI) [1], prostate biopsy procedures directly targeting suspicious MRI lesions are increasingly performed. These biopsies can be guided either in bore, cognitively during a transrectal ultrasound (TRUS) procedure, or using an MRI-TRUS fusion strategy in which the MRI-derived lesion locations have to be coregistered to real-time TRUS imaging based on the prostatic contours $[2,3]$. A recent meta-analysis showed a comparable significant prostate cancer detection rate for the in-bore, fusion, and cognitive guidance strategies, while in-bore strategy had an advantage over cognitive guidance in overall prostate cancer detection [4]. Nevertheless, direct in-bore biopsy is restrained in terms of time and cost involved [5]. On the contrary, TRUS prostate images have to be delineated (semi-)manually in current MRI-TRUS fusion systems, which use the outer contours as a landmark for registration. This is also time consuming, operator dependent, and error prone [6]. As a matter of fact, the laboriousness and cost of MRI-TRUS fusion are often considered key aspects hampering the clinical adoption of MRI-targeted procedures [2].

The introduction of computer-driven segmentation would drastically minimise the required user input, provided that the algorithm is fully automated and real time. Although substantial effort has been invested in (semi-) automatic segmentation of the prostate over the past decades [7,8], it remains difficult to obtain reliable and generalisable results in TRUS acquisitions [9]. Even more challenging is zonal segmentation, that is, the separation of the peripheral zone (PZ) and the transition zone (TZ) [10]. The glandular tissue of the posteriorly located PZ is histologically distinctly different from that of the anterior TZ, and also the central zone and anterior fibromuscular stroma (AFS), which for this work will be segmented as a whole. We do not assess the central zone and AFS as separate entities for segmentation, since delineating the zonal anatomy of these regions is subject to high interuser variability, while clinical implication for prostate cancer diagnosis (eg, through multiparametric MRI) is often evaluated for the $\mathrm{PZ}$ and the $\mathrm{TZ}$ (in combination with the remaining zones) only. Although zonal segmentation is not directly necessary, zonal boundary could be a valuable landmark for MRI-TRUS registration. Moreover, it is very useful for other applications of segmentation (eg, zonal volume estimation, guidance in needle positioning, and focal treatment planning). Very recently, deep-learning strategies were introduced using datasets with a limited number of patients
$[11,12]$. Yet, the presented methods did not contain zonal segmentation, and as deep-learning results tend to be poorly generalisable to different acquisition systems, it remains unclear how well the methods translate to other ultrasound scanners.

In this study, we develop and validate a scanner-adaptable deep-learning network architecture for zonal classification. We consecutively demonstrate its ability to segment in real time, accurately classify prostatic zones, adapt to different scanners and operators, and evaluate its own percase reliability.

\section{Patients and methods}

\subsection{Patient selection}

To develop and validate the segmentation algorithm, three separate datasets were analysed. A 198-image "Philips" dataset was obtained in 78 patients at the Academic Medical Center in Amsterdam using an iU22 ultrasound scanner (Philips Healthcare, Bothell, WA, USA) equipped with either a $\mathrm{C} 10-3 \mathrm{v}$ or a $\mathrm{C} 8-4 \mathrm{v}$ probe. Analogously, a "BK Medical" dataset was acquired at the Jeroen Bosch Hospital in "sHertogenbosch using a Pro Focus 2202 device and a Prostate Triplane 8818 ultrasound probe. A total of 84 images from 55 patients were included. Finally, 144 images from 48 patients comprising a "SuperSonic Imagine" dataset were collected at the Martini Klinik at the University Medical Center Hamburg-Eppendorf using an Aixplorer (SuperSonic Imagine, Aix-en-Provence, France) scanner with an SE12-3 transducer. The TRUS fundamental view in side-byside contrast mode recordings was made available to us. All patients signed an informed consent form, and the acquisitions were approved by the institutional review boards. The device and clinical characteristics of these datasets are listed in Table 1.

\subsection{Data annotation}

For the "Philips" and "BK Medical" datasets, the outer and zonal contours of TRUS acquisition were annotated in consensus by a five-person expert panel, consisting of three urologists (C.M., A.P., and M.G.) with $>4 \mathrm{yr}$ of experience and two engineers (R.S. and R.W.) familiar with TRUS images for $>3$ yr. For the "SuperSonic Imagine" dataset, the (zonal) contours were delineated in consensus by two experts (C. M. and R.W.). Edges between the hypoechogenic prostate tissue and the more hyperechogenic surroundings as well as the nonechogenic bladder served as visual cues for the manual delineation $[13,14]$. 
Table 1 - Scanner specifications and demographic breakdown of datasets.

\begin{tabular}{|c|c|c|c|}
\hline Dataset & Philips & BK Medical & SuperSonic Imagine \\
\hline Manufacturer & Philips HealthCare & BK Medical & SuperSonic Imagine \\
\hline Scanner & iU22 & Pro Focus 2202 & Aixplorer \\
\hline Probe & $\mathrm{C} 10-3 \mathrm{~V}$ and $\mathrm{C} 8-4 \mathrm{v}$ & Prost. Triplane 8818 & SE12-3 \\
\hline Side-by-side view with & Contrast & Contrast & Contrast \\
\hline Transmit frequency $(\mathrm{MHz})$ & 3.5 & 4 & 3.2 \\
\hline Frame rate $(\mathrm{Hz})$ & 9 & 9 & 27 \\
\hline Average pixel spacing (mm) & 0.17 & 0.16 & 0.10 \\
\hline Number of images & 198 & 94 & 144 \\
\hline Number of patients & 78 & 55 & 48 \\
\hline Images per patient & $1-4$ & $1-2$ & 3 \\
\hline Age (yr), median (range) & $62(37-83)$ & $65(49-74)$ & $65(45-77)$ \\
\hline Prostate volume $(\mathrm{ml})$, median & 37 & 35 & 40 \\
\hline \multicolumn{4}{|l|}{ Biopsy Gleason score, $n$ (\%) } \\
\hline $3+3=6$ & $37(47.4)$ & $30(54.5)$ & $9(18.8)$ \\
\hline $3+4,4+3=7$ & $32(41.1)$ & $19(34.6)$ & $29(60.4)$ \\
\hline$\geq 4+4=8$ & $9(11.5)$ & $6(10.9)$ & $10(20.8)$ \\
\hline
\end{tabular}

\subsection{Deep learning}

In this work, we propose a method based on deep learning, which is a neural-network approach in which images are described by a hierarchy of semantic features allowing pixelwise classification. We designed a fully convolutional neural network to map input TRUS images to output segmentations, an algorithm consisting of multiple nonlinear mappings (layers) that extract a hierarchy of representations at a plurality of scales. The main architecture is based on a particular widely adopted network topology, the "U-net" [15]. First, the algorithm encodes the TRUS images into a latent feature space through a series of convolutional and subsampling (ie, pooling) layers, after which it decodes this feature space into a categorically segmented output through an equivalent number of "deconvolution" layers. In total, the network is 14 convolutional layers deep, with almost 1 million trainable parameters. A Softmax function is subsequently applied to yield pixel-wise class probabilities. We used skip connections between the encoder and the decoder [16], and employed batch normalisation to accelerate training convergence [17].

\subsection{Data augmentation}

We promote convergence toward model parameterisation that is invariant to several common variations in TRUS images, through an aggressive and extensive online dataaugmentation strategy. For each iteration, $60 \%$ of the imageannotation pairs are heavily processed by applying random amounts of translation, rotation, scaling, shearing, elastic warping, cropping, blurring, contrast alterations, noise addition, and mirroring in the anterior-posterior axis [15].

\subsection{Supervised domain adaptation}

While data augmentation enables learning of a network that generalises well across TRUS images of different patients obtained in similar settings, it may fall short when employed on images obtained using an entirely different ultrasound system. We therefore exploit a supervised adaptation strategy by which we adapt the network to a new "domain" by including a small number of annotated training examples from this new domain.

\subsection{Segmentation confidence}

Furthermore, we assess the confidence of each segmentation by a Bayesian 40-fold Monte Carlo dropout approach; we define areas with segmentation disconcordance to be of lower confidence.

\subsection{Conventional method}

As an example of a conventional model-based segmentation method, we tailored an edge-detection-based segmentation algorithm to our grey-scale acquisitions based on the works of Pathak et al [18] and Gong et al [19]. This method will serve as the benchmark method for automated segmentation.

\subsection{Model validation}

Prior to analysis, the "Philips" dataset was divided in a 54patient training set and a 24-patient test set. To analyse the "BK Medical" and "SuperSonic Imagine" sets, all patients in the "Philips" set were used for training and the first 10 patients of each set were selected for the supervised domain adaptation. Image segmentations were subsequently compared with the manual delineations in terms of accuracy (ie, number of correctly-classified pixels compared with the total number of pixels in the image) and the Jaccard index [20], a measure for region overlap ranging from 0 (worst) to 1 (best), alternatively referred to as the intersection over union. The Jaccard index is the ratio between the shared and the total surface of the two shapes. The maximum absolute distance between the delineations (ie, Hausdorff distance [20]) was used to quantify the occurrence of local unexpected deviations. In addition, we test the image mean segmentation confidence as a measure of the overall segmentation quality. 


\subsection{Statistical analysis}

As most performance measures were not normally distributed (as assessed by a $\chi^{2}$ normality test), the level of significance to compare differences within a dataset was verified using a Wilcoxon signed rank test. Between datasets, the level of significance was verified using the Wilcoxon rank sum test. Any $p$ value below 0.05 is considered significant. Unless otherwise stated, we refer to the median value and its 5-95\% confidence interval.

\section{Results}

\subsection{Prostate segmentation}

In the "Philips" test set of 44 images (from the last 24 patients), the deep-learning approach yielded improved accuracy of 98\% (95-99\%) compared with 95\% (86-98\%; $p<$ 0.001 ) for the conventional segmentation method and an improved Jaccard index of 0.93 (0.80-0.95) compared with 0.81 (0.53-0.92; $p<0.001$; Fig. 1). The Hausdorff distance per image was diminished to 2.99 (1.29-8.36) $\mathrm{mm}$ from 7.60 (2.59-19.79) $\mathrm{mm}(p<0.001)$. Figure 2 shows five examples reflecting that, although the conventional method generally generated good-quality whole-gland segmentations, its performance was poor in low-contrast images, images containing bright calcifications, and images with shadow artefacts. The deep-learning approach was much more stable, showing only diminished performance in images with unusual structures (eg, large cysts, extracapsular tumour growth) or large shadow artefacts (see also the Segmentation confidence section). Moreover, the segmentation time is suitable for real-time applications, being $0.0129 \pm 0.0007 \mathrm{~s}$ instead of $11.2 \pm 0.5 \mathrm{~s}$ per image.

\subsection{Zonal classification}

To our knowledge, no algorithm for automatic zonal segmentation in TRUS has ever been proposed, as the zonal boundary is often less pronounced than the glandular boundary or obscured by calcifications. Indeed, our zonal segmentation performance was lower than that of the gland contour delineation (Table 2). Since the PZ is often smaller, its delineation was generally less accurate. As some typical examples in Figure 2 demonstrate, zonal classification was fairly stable. For the envisaged applications in biopsy-needle placement, focal treatment planning, or evaluation of other TRUS modalities, as further discussed in the Discussion section, this degree of accuracy may be considered sufficient.

\subsection{Scanner-adapted segmentation}

In deep learning, classification performance is strongly dependent on the representativeness of the cases present

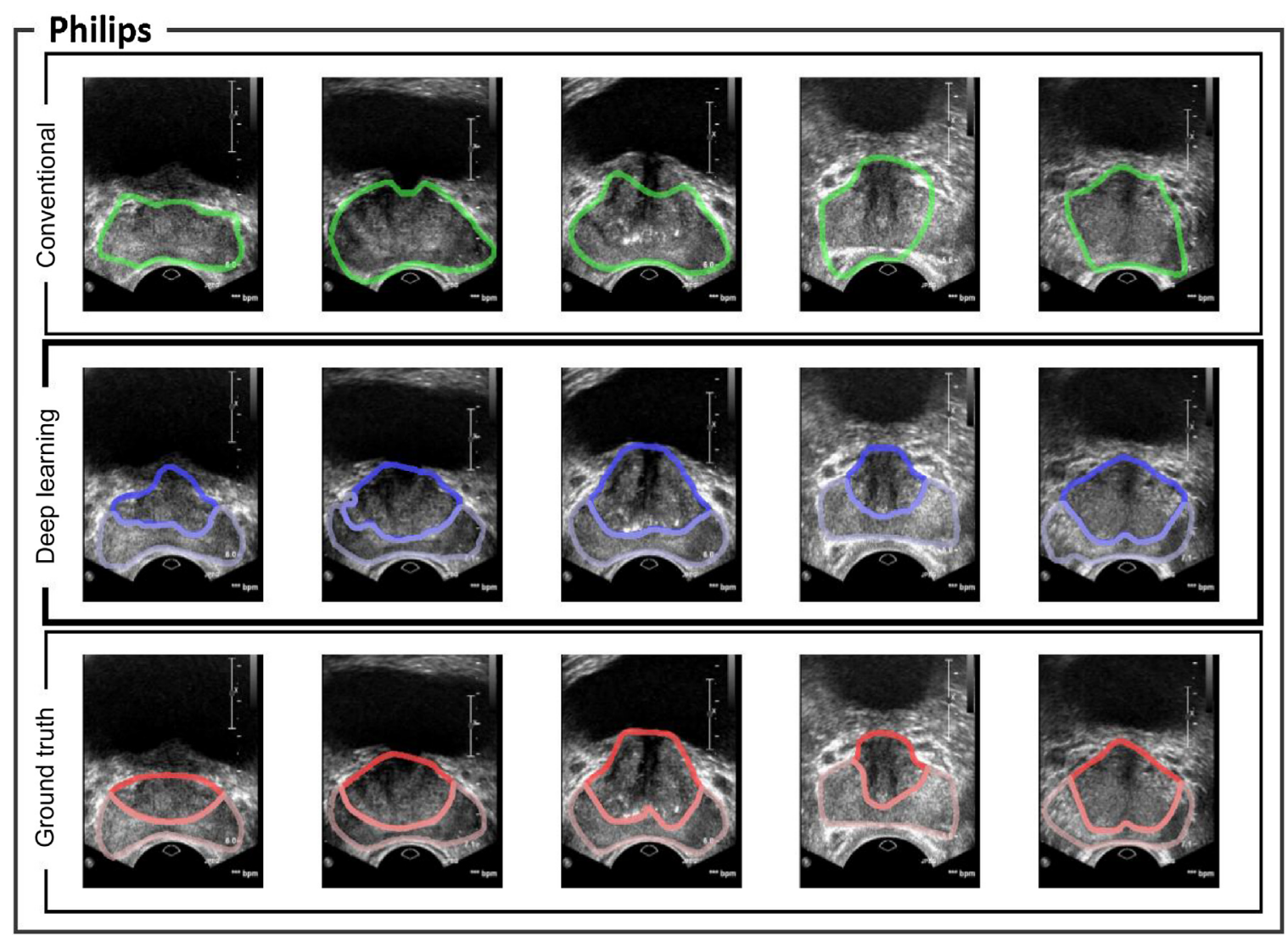

Fig. 1 - Five examples of B-mode images from the "Philips" dataset of prostatic segmentation by a conventional algorithm, the proposed deep-learning approach, and expert annotations. 

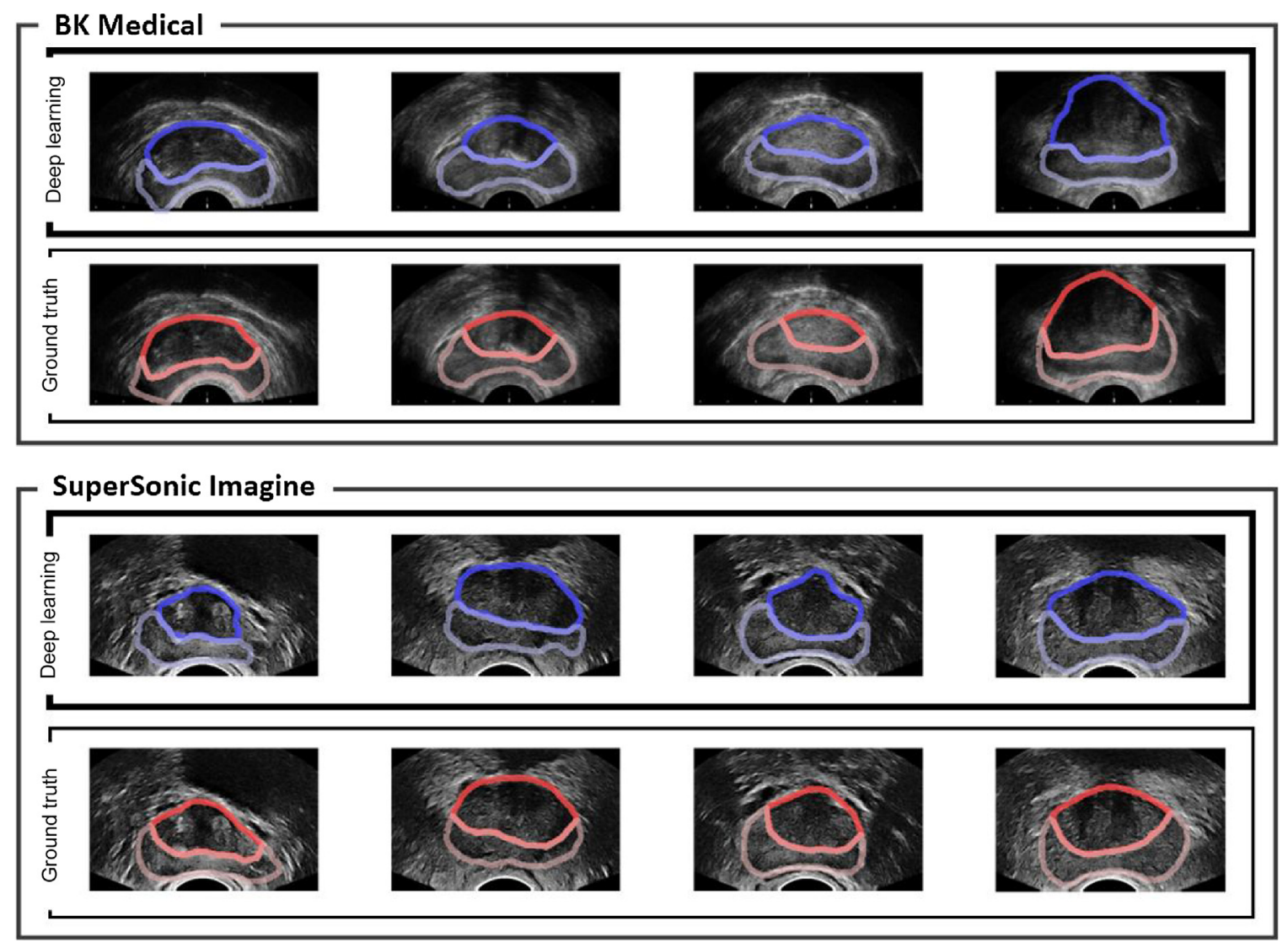

Fig. 2 - Examples of B-mode images from the "BK Medical" and "SuperSonic" datasets with segmentation by the proposed deep-learning approach and by experts.

Table 2 - Segmentation performance in comparison with conventional technique.

\begin{tabular}{llcc}
\hline Segmentation method & $\begin{array}{c}\text { Pixel-wise accuracy }(\%) \\
\text { Med }(\mathrm{CI})\end{array}$ & $\begin{array}{c}\text { Jaccard index } \\
\text { Med }(\mathrm{CI})\end{array}$ & $\begin{array}{c}\text { Hausdorff distance (mm) } \\
\text { Med }(\mathrm{CI})\end{array}$ \\
\hline Conventional method & $95(86-98)$ & $0.81(0.53-0.92)$ & $7.8(2.6-20)$ \\
Proposed method & $98(95-99)$ & $0.93(0.80-0.96)$ & $3.0(1.3-8.7)$ \\
Peripheral zone & $97(95-99)$ & $0.75(0.44-0.87)$ & $5.1(2.3-18)$ \\
Transition zone & $98(96-99)$ & $0.82(0.65-0.93)$ & $4.6(2.2-7.9)$ \\
\hline $\mathrm{Cl}=$ confidence interval; Med = median. & & & \\
\hline
\end{tabular}

in the training set. Therefore, using only the "Philips" dataset, which differs in, for example, brightness, resolution, and image formation from other devices, prostate segmentation performance was significantly lower when directly applied to other datasets (Table 3). However, after supervised domain adaptation, performance in the test set consisting of the remaining 45 and 38 patients in the "BK Medical" and "SuperSonic Imagine" datasets was restored; the Hausdorff distances (ie, the most discriminative measure) did not differ significantly from those achieved in the original

Table 3 - Scanner-adapted prostate segmentation performance for the separate datasets.

\begin{tabular}{|c|c|c|c|c|c|}
\hline Test set from & Training set from & $\begin{array}{c}\text { Pixel-wise accuracy (\%) } \\
\text { Med }(\mathrm{CI})\end{array}$ & $\begin{array}{l}\text { Jaccard index } \\
\text { Med }(\mathrm{CI})\end{array}$ & $\begin{array}{c}\text { Hausdorff distance }(\mathrm{mm}) \\
\operatorname{Med}(\mathrm{CI})\end{array}$ & $p$ value \\
\hline Philips & Philips & 98 (95-99) & $0.93(0.80-0.96)$ & $3.0(1.3-8.7)$ & 1 \\
\hline \multirow[t]{2}{*}{ BK Medical } & Philips & $96(89-98)$ & $0.86(0.57-0.94)$ & $5.4(1.7-16)$ & $2.6 \times 10^{-4}$ \\
\hline & Philips + sDA & 97 (91-98) & $0.90(0.69-0.94)$ & $3.4(1.7-9.2)$ & 0.37 \\
\hline \multirow{2}{*}{ SuperSonic Imagine } & Philips & 97 (90-99) & $0.91(0.72-0.95)$ & $3.9(1.4-15)$ & 0.036 \\
\hline & Philips + sDA & 98 (95-99) & $0.93(0.85-0.96)$ & $2.8(1.1-9.4)$ & 0.54 \\
\hline
\end{tabular}



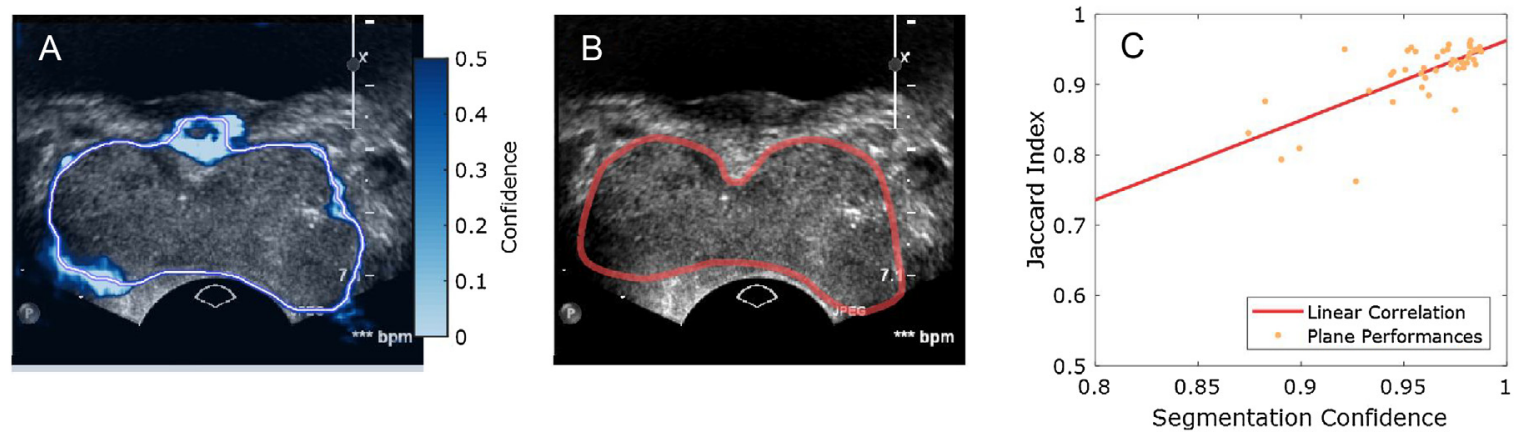

Fig. 3 - Example of (A) prostatic segmentation with associated confidence map, with the lower-confidence pixels depicted in white to blue, (B) the expert ground truth, and $(C)$ a plot of the relation between segmentation confidence and the Jaccard index in the "Philips" dataset.

domain (ie, Philips scanner). For the "Philips," "BK Medical," and "SuperSonic Imagine," we obtained Hausdorff distances of 3.0, $3.4(p=0.37)$, and $2.8 \mathrm{~mm}(p=0.54)$, respectively; Figure 3 exemplifies these results.

\subsection{Segmentation confidence}

An example of a segmentation confidence map is depicted in Figure 3A, highlighting low-confidence areas in blue to white. The confidence map accurately accentuates the regions where prostatic segmentation deviated from the ground truth due to a shadowing artefact (posterior left) and an unusual shape (anteriorly). As shown in Figure 3C, there is a strong correlation between the image mean confidence and the corresponding Jaccard index (linear Pearson correlation coefficient of $0.72, p<0.001$ ). This measure could therefore be used to swiftly identify a potentially erroneous result.

\section{Discussion}

Owing to its ability to detect significant prostate cancer while reducing overdetection of indolent disease, targeted biopsy of MRI suspicious lesions is rapidly becoming common practice in the diagnostic pathway [1]. Different MRITRUS fusion platforms have been developed to facilitate the needle guidance on real-time TRUS imaging, as cognitive biopsy is likely dependent on the experience and skill of the operator [21].

Automatic, real-time, and robust segmentations of the prostate and its main zones on TRUS imaging have great value for MRI-TRUS fusion technology and may help in the adoption of such technique in clinical practice, as real-time on-line segmentation alleviates the need for repeated manual delineation. TRUS is often performed freehand, is susceptible to (speckle) noise, contains prostatic boundaries that are poorly visible or obscured by (shadow) artefacts [22], and exhibits large variations in image quality and appearance between operators and scanner manufacturers. Therefore, the robustness of the method was studied in large and highly varied patient cohorts, showing high accuracy and generalisability.

A limitation of the current study is the reliability of the ground truth. Even though we employed a five-person consensus panel of experts, manual prostatic segmentation-and, to a higher extent, zonal segmentation-is notoriously subject to interuser variability [23]. The use of consensus delineations rather than those of a single observer, however, enabled us to incorporate the shared vision of all experts to train the segmentation algorithm. Another limiting aspect relates to the dataset consisting of patients with known prostate cancer, which may exhibit an abnormal TRUS appearance [24]. On the contrary, segmentation in this group is likely to be more difficult than in a healthy population, and can be considered more representative for patients undergoing TRUS biopsy. Although the test-set results presented in this study demonstrate the generalisability of the algorithm, a follow-up in a larger cohort of patients referred for imaging-targeted biopsy is still needed to establish this more definitively. Furthermore, as the fundamental mode in the side-by-side contrast view is generally of lower quality than the full B mode, our datasets represent the worst-case scenario to the segmentation algorithm.

The proposed approach achieves a similar degree of accuracy to prostate segmentation on MRI [25,26], even though TRUS segmentation is more challenging with cognitive interobserver variability of already around $10 \%$ in the prostate surface area [23]. It has been established that a technical registration error in the millimetre range can be considered sufficient for targeting a high-grade hotspot [27]. Provided that prostate fusion systems are appreciably accurate, this deep-learning approach can aid in the fusion technology to attain the required level of precision.

Apart from its value for fast and more accurate MRI-TRUS fusion, automatic (zonal) prostate segmentation could also be used for a more accurate and quick assessment of the gland's volume [22], which is often reported to be operator dependent, difficult to reproduce, and of limited accuracy [28]. Real-time segmentation could also assist the urologist in needle positioning during biopsy [29]. Moreover, zonal and prostate segmentations are of utmost importance for planning (focal) therapy, such as brachytherapy [30] and high-intensity focused ultrasound [31], to clearly mark the capsule of the prostate in order to spare the tissue beyond that point. Moreover, it might also aid in the reviewing of elastography images or contrast-enhanced ultrasound videos in patients with a suspicion of prostate cancer. 
The $\mathrm{TZ}$ is much stiffer than the $\mathrm{PZ}$ and thus requires a different cut-off elasticity for lesions to be suspicious [32]; similarly, the influx of contrast agents has been reported to be different in the two zones [33]. Finally, this algorithm could well be combined with artificial-intelligence applications to analyse ultrasound images for the presence of prostate cancer, such as C-TRUS or quantitative ultrasound [34-36], potentially allowing for ultrasoundbased rather than MRI-based targeted biopsy [37-39].

In the future, it would be of interest to investigate the effect of automatic, real-time segmentation on the reliability of the fusion biopsy procedure. As in-bore biopsy requires specialised equipment yielding comparable results $[3,4]$, an easy-to-use, reliable fusion system could facilitate more rapid uptake of MRI-guided biopsy in the clinic.

\section{Conclusions}

Prostate segmentation in TRUS B-mode images is challenging and has been studied extensively because of its clinical relevance, for example, for MRI-TRUS fusion biopsy. We presented a deep-learning-based approach for fully automatic (zonal) prostate segmentation and demonstrated its speed, reliability, and ability to adapt to different scanners on a large set of TRUS images from different institutions. Given the image variety in the current datasets, we expect that this adaptation ability can be extrapolated to other manufacturers.

Author contributions: Ruud J.G. van Sloun had full access to all the data in the study and takes responsibility for the integrity of the data and the accuracy of the data analysis.

Study concept and design: van Sloun, Wildeboer.

Acquisition of data: Mannaerts, Postema, Gayet.

Analysis and interpretation of data: van Sloun, Wildeboer.

Drafting of the manuscript: van Sloun, Wildeboer.

Critical revision of the manuscript for important intellectual content: All authors.

Statistical analysis: van Sloun, Wildeboer.

Obtaining funding: Salomon, Wijkstra, Beerlage, Mischi.

Administrative, technical, or material support: None.

Supervision: Salomon, Wijkstra, Beerlage, Mischi.

Other: None.

Financial disclosures: Ruud J.G. van Sloun certifies that all conflicts of interest, including specific financial interests and relationships and affiliations relevant to the subject matter or materials discussed in the manuscript (eg, employment/affiliation, grants or funding, consultancies, honoraria, stock ownership or options, expert testimony, royalties, or patents filed, received, or pending), are the following: Hessel Wijkstra: unrestricted grant from Dutch Cancer Society (\#UVA2013-5941). Massimo Mischi: European Research Council Starting Grant (\#280209). Maudy Gayet: research grant from Astellas Pharma Netherlands B.V. Rogier R. Wildeboer: IMPULS2-pogram within the Eindhoven University of Technology in collaboration with Philips.

Funding/Support and role of the sponsor: The authors would like to acknowledge NVIDIA Corporation for granting the Titan XP GPU processor.

\section{References}

[1] Kasivisvanathan V, Rannikko ASAS, Borghi M, et al. MRI-targeted or standard biopsy for prostate-cancer diagnosis. N Engl J Med 2018;378:1767-77.

[2] Tyson MD, Arora SS, Scarpato KR, Barocas D. Magnetic resonanceultrasound fusion prostate biopsy in the diagnosis of prostate cancer. Urol Oncol Semin Orig Investig 2018;34:326-32.

[3] Kuru TH, Herden J, Zugor V, et al. How to perform image-guided prostate biopsy: in-bore and fusion approaches. Eur Urol Focus 2016;2:151-3.

[4] Wegelin O, van Melick HHE, Hooft L, et al. Comparing three different techniques for magnetic resonance imaging-targeted prostate biopsies: a systematic review of in-bore versus magnetic resonance imaging-transrectal ultrasound fusion versus cognitive registration. Is there a preferred technique? Eur Urol 2017;71:517-31.

[5] Venderink W, Govers TM, de Rooij M, Fütterer JJ, Sedelaar JPM. Costeffectiveness comparison of imaging-guided prostate biopsy techniques: systematic transrectal ultrasound, direct in-bore MRI, and image fusion. Am J Roentgenol 2017;208:1058-63.

[6] Logan JK, Rais Bahrami S, Turkbey B, et al. Current status of magnetic resonance imaging (MRI) and ultrasonography fusion software platforms for guidance of prostate biopsies. BJU Int 2013;114:641-52.

[7] Ghose S, Oliver A, Martí R, et al. A survey of prostate segmentation methodologies in ultrasound, magnetic resonance and computed tomography images. Comput Methods Programs Biomed 2012;108:262-87.

[8] Singh RP, Gupta S, Acharya UR. Segmentation of prostate contours for automated diagnosis using ultrasound images: A survey. J Comput Sci 2017;21:223-31.

[9] Kamnitsas K, Baumgartner C, Ledig C, et al. Unsupervised domain adaptation in brain lesion segmentation with adversarial networksInternational Conference on Information Processing in Medical Imaging. 2017;597-609.

[10] McNeal JE. The zonal anatomy of the prostate. Prostate 1981;2:3549.

[11] Anas EMA, Mousavi P, Abolmaesumi P. A deep learning approach for real time prostate segmentation in freehand ultrasound guided biopsy. Med Image Anal 2018;48:107-16.

[12] Ghavami N, Hu Y, Bonmati E, et al. Automatic slice segmentation of intraoperative transrectal ultrasound images using convolutional neural networks. Proc SPIE Med Imaging Image Guided Proced Robot Interv Model 2018;10576:1057603.

[13] Villers A, Terris MK, McNeal JE, Stamey TA. Ultrasound anatomy of the prostate: the normal gland and anatomical variations. J Urol 1990; $143: 732-8$.

[14] Watson LR. Ultrasound anatomy for prostate brachytherapy. Semin Surg Oncol 1997;13:391-8.

[15] Ronneberger O, Fischer P, Brox T. U-Net: convolutional Networks for biomedical image segmentationInternational Conference on Medical Image Computing and Computer-assisted Intervention. Cham, Switzerland: Springer; 2015. p. 234-41.

[16] Mao X, Shen C, Yang Y-B. Image restoration using very deep convolutional encoder-decoder networks with symmetric skip connections. Adv Neural Inf Process Syst 2016;2802-10.

[17] Ioffe S, Szegedy C. Batch normalization: accelerating deep network training by reducing internal covariate shiftIn: Proceedings of the 32nd International Conference on Machine Learning. $2015 ; 1-9$

[18] Pathak SD, Haynor DR, Kim Y. Edge-guided boundary delineation in prostate ultrasound images. IEEE Trans Med Imaging 2000;19:1211-9. 
[19] Gong L, Pathak SD, Haynor DR, Cho PS, Kim Y. Parametric shape modeling using deformable superellipses for prostate segmentation. IEEE Trans Med Imaging 2004;23:340-9.

[20] Taha AA, Hanbury A. Metrics for evaluating 3D medical image segmentation: analysis, selection, and tool. BMC Med Imaging 2015;15:29.

[21] Warlick C, Futterer J, Maruf M, et al. Beyond transrectal ultrasoundguided prostate biopsies: available techniques and approaches. World J Urol 2019;37:419-27.

[22] Aarnink R, Pathak SD, de la Rosette JJMCH, Debruyne FMJ, Kim Y, Wijkstra H. Edge detection in prostatic ultrasound images using integrated edge maps. Ultrasonics 1998;36:635-42.

[23] Sandhu GK, Dunscombe P, Meyer T, Pavamani S, Khan R. Inter- and intra-observer variability in prostate definition with tissue harmonic and brightness mode imaging. Int J Radiat Oncol 2012;82:e9-16.

[24] Harvey CJ, Pilcher J, Richenberg J, Patel U, Frauscher F. Applications of transrectal ultrasound in prostate cancer. Br J Radiol 2012;85:S3-17.

[25] Litjens G, Toth R, van de Ven W, et al. Evaluation of prostate segmentation algorithms for MRI: the PROMISE12 challenge. Med Image Anal 2014;18:359-73.

[26] Tian Z, Liu L, Zhang Z, Fei B. PSNet: prostate segmentation on MRI based on a convolutional neural network. J Med Imaging 2018;5:21206-8.

[27] van de Ven WJM, Hulsbergen-van de Kaa CA, Hambrock T, Barentsz JO, Huisman HJ. Simulated required accuracy of image registration tools for targeting high-grade cancer components with prostate biopsies. Eur Radiol 2013;23:1401-7.

[28] Bazinet M, Karakiewicz PI, Aprikian AG, et al. Reassessment of nonplanimetric transrectal ultrasound prostate volume estimates. Urology 1996;47:857-62.

[29] Jeffrey B, Derek C, Lori G, et al. Mechanically assisted 3D ultrasound guided prostate biopsy system. Med Phys 2008;35:5397-410.
[30] Hong CW, Reddy CA, Wilkinson A, Klein EA, Ciezki JP. Original paper Influence of zonal dosimetry on prostate brachytherapy outcomes. J Contemp Brachyther 2015;7:17-22.

[31] Chiang PH, Liu YY. Comparisons of oncological and functional outcomes among radical retropubic prostatectomy, high dose rate brachytherapy, cryoablation and high-intensity focused ultrasound for localized prostate cancer. Springerplus 2016;5:1905.

[32] Rouvière O, Melodelima C, Hoang Dinh A, et al. Stiffness of benign and malignant prostate tissue measured by shear-wave elastography: a preliminary study. Eur Radiol 2017;27:1858-66.

[33] Halpern EJ. Contrast-enhanced ultrasound imaging of prostate cancer. Rev Urol 2006;8:S29.

[34] Loch T, Leuschner I, Genberg C, et al. Artificial neural network analysis (ANNA) of prostatic transrectal ultrasound. Prostate 1999;39:198-204.

[35] Feleppa EJ, Ennis RD, Schiff PB, et al. Spectrum-analysis and neural networks for imaging to detect and treat prostate cancer. Ultrason Imaging 2001;23:135-46.

[36] Wildeboer RR, Postema AW, Demi L, Kuenen MPJ, Wijkstra H, Mischi M. Multiparametric dynamic contrast-enhanced ultrasound imaging of prostate cancer. Eur Radiol 2017;27:3226-34.

[37] Gill IS, de Castro Abreu AL, Palmer S, et al. Trans-rectal ultrasound visibility of prostate lesions identified by magnetic resonance imaging increases accuracy of image-fusion targeted biopsies. World J Urol 2015;33:1669-76.

[38] Loch T, Grabski B, Bäurle L, Tokas T, Paul U. A 12-year follow-up of ANNA/C-TRUS image-targeted biopsies in patients suspicious for prostate cancer. World J Urol 2017;36:699-704.

[39] Müller S, Lilleaasen G, Sand TE, et al. Poor reproducibility of PIRADS score in two multiparametric MRIs before biopsy in men with elevated PSA. World J Urol 2018;36:687-91. 